\title{
Properties of Spatial Cox Process Models
}

\author{
J. Møller
}

Aalborg University

\begin{abstract}
Probabilistic properties of Cox processes of relevance for statistical modelling and inference are studied. Particularly, we study the most important classes of Cox processes, including log Gaussian Cox processes, shot noise Cox processes, and permanent Cox processes. We consider moment properties and point process operations such as thinning, displacements, and superpositioning. We also discuss how to simulate specific Cox processes.
\end{abstract}

Keywords. Doubly stochastic process; edge effects; intensity; log Gaussian Cox process; mixed Poisson process; pair correlation function; permanent process; random displacements; shot noise Cox process; simulation, spatial point process; superposition; thinning.

\section{Introduction}

Spatial point pattern datasets occur in a variety of sciences, and the theory of spatial point processes and their applications have been treated in various textbooks, including Stoyan, Kendall \& Mecke (1995), Lieshout (2000), Diggle (2003), Møller \& Waagepetersen (2003), and Baddeley, Gregori, Mateu, Stoica \& Stoyan (2006). Indeed this is an active research area, and the purpose of the present paper is to discuss recent advances in a special topic, namely spatial Cox process theory. A Cox process $\mathbf{X}$ on the $d$-dimensional Euclidean space $\mathbb{R}^{d}$ results from a doubly stochastic process given by a random non-negative function $\boldsymbol{\Lambda}=(\Lambda(u))_{u \in \mathbb{R}^{d}}$ such that $\mathbf{X}$ conditional on $\boldsymbol{\Lambda}$ is a Poisson process with intensity function $\boldsymbol{\Lambda}$. The Cox process can also be specified by the random 
intensity measure $M(B)=\int_{B} \Lambda(u) \mathrm{d} u$, and it should be obvious that many of the definitions and results in this paper easily extend to a Cox process driven by a random measure which is not necessarily absolutely continuous with respect to Lebesgue measure. Cox processes constitute the most useful class of spatial point process models for aggregated point patterns, where the statistical aspects are studied in the above-mentioned textbooks and in Møller \& Waagepetersen (2006), while probabilistic aspects are studied in Grandell (1976), Kingman (1993) and Daley \& Vere-Jones (2003).

This paper focuses on the probabilistic properties of Cox processes which are important for statistical modelling and inference. Section 2 provides some background material on spatial point processes and particularly the Poisson process. Due to its mathematical tractability, the Poisson process has enjoyed popularity out of proportion to its realism. In Section 3, we therefore introduce Cox processes and study some of their general properties. The main section is Section 4, where we study the most important classes of Cox processes, namely log Gaussian Cox processes (Møller, Syversveen \& Waagepetersen, 1998) and shot noise Cox processes (Brix, 1999 and Møller, 2003) together with mixed Poisson processes and also a new class of models called permanent Cox processes (McCullagh \& Møller, 2005). In particular, we consider moment properties and point process operations such as thinning, displacements, and superpositioning. We also consider how to simulate specific Cox processes, since simulation is an indispensable tool for statistical inference (Møller \& Waagepetersen, 2003 and 2006).

\section{Preliminaries}

\subsection{Fundamental Definitions}

For simplicity and ease of presentation, we define a spatial point process on $\mathbb{R}^{d}$ as a locally finite random set $\mathbf{X} \subset \mathbb{R}^{d}$, meaning that for any bounded Borel set $B \subset \mathbb{R}^{2}$, the number of points in $\mathbf{X}_{B}=\mathbf{X} \cap B$ is a finite random variable denoted $N(B)$. This definition is illuminating and sufficient for most applications, and the extension of this and other definitions in this paper to point processes with multiple points is rather straightforward (viz. by viewing the counts $N(B)$ as a counting process).

We say that $\mathbf{X}$ is stationary respective isotropic if its distribution is invariant under translations in $\mathbb{R}^{d}$ respective rotations about the origin in $\mathbb{R}^{d}$. Stationarity and isotropy may be reasonable assumptions for point processes observed within a homogeneous study region, but it is important to evaluate these assumptions (Baddeley, Møller \& Waagepetersen, 2000, Baddeley, Turner, Møller \& Hazelton, 2005 and Møller \& Waagepetersen, 2006). We shall later consider 
inhomogeneous point process models which incorporate spatial covariate information.

For integers $n \geqslant 1$, the $n$th order factorial moment measure $\mu^{(n)}$ of $\mathbf{X}$ is defined by

$$
\mu^{(n)}(A)=\mathrm{E} \sum_{u_{1}, \ldots, u_{n} \in \mathbf{X}}^{\neq} \mathbf{1}\left[\left(u_{1}, \ldots, u_{n}\right) \in A\right]
$$

for Borel sets $A \subseteq \mathbb{R}^{d n}$, where $\neq$ over the summation sign means that the sum runs over all pairwise different points $u_{1}, \ldots, u_{n}$ in $\mathbf{X}$, and $\mathbf{1}[\cdot]$ is the indicator function. Throughout this paper we assume that $\mu^{(n)}$ is locally finite and has a density $\rho^{(n)}$ with respect to Lebesgue measure on $\mathbb{R}^{d n}$, where we call $\rho^{(n)}$ the $n$th order product density. Intuitively, if $u_{1}, \ldots, u_{n} \in \mathbb{R}^{d}$ are pairwise different, $\rho^{(n)}\left(u_{1}, \ldots, u_{n}\right) \mathrm{d} u_{1} \cdots \mathrm{d} u_{n}$ is the probability of observing $n$ points from $\mathbf{X}$ occurring jointly in each of $n$ infinitesimally small regions of areas $\mathrm{d} u_{1}, \ldots, \mathrm{d} u_{n}$ and containing $u_{1}, \ldots, u_{n}$.

The first and second order factorial moment measures are most important for statistical inference, see e.g. Møller \& Waagepetersen (2003 and 2006). In particular, $\mu(A)=\mu^{(1)}(A)=\operatorname{EN}(A)$ is the moment measure, and $\rho=\rho^{(1)}$ is the intensity (function). The pair correlation function is defined by

$$
g(u, v)=\frac{\rho^{(2)}(u, v)}{\rho(u) \rho(v)}
$$

(provided $\rho(u)>0$ and $\rho(v)>0$ ). This kind of normalizing is useful, since $g \equiv 1$ in the case of no interaction (see Section 2.3). We interpret $g(u, v)>1$ as attraction between points of the process at locations $u$ and $v$, and $g(u, v)<1$ as repulsion at the two locations. Stationarity of $\mathbf{X}$ implies that we can assume that $\rho(u)$ is constant and $g(u, v)=g(u-v)$ is translation invariant, and if also isotropy holds then $g(u, v)=g(\|u-v\|)$ depends only on the distance between $u$ and $v$.

\subsection{Point Process Operations}

We shall study three fundamental operations for spatial point processes.

First, suppose $\pi: \mathbb{R}^{d} \mapsto[0,1]$ is a Borel function. An independent $\pi$ thinning of $\mathbf{X}$ is obtained by independent retaining each point $u$ in $\mathbf{X}$ with probability $\pi(u)$. The intensity of the thinned process is $\pi(u) \rho(u)$, while $g$ is the same for the two processes.

Second, for points $u$ in $\mathbf{X}$, suppose $m_{u}$ is a random variable in $\mathbb{R}^{d}$ with density $p_{u}$ with respect to Lebesgue measure, where conditional on $\mathbf{X}$, the $m_{u}$ are independent. Then the point process $\mathbf{X}^{*}=\left\{u+m_{u}: u \in \mathbf{X}\right\}$ is produced 
by random independent displacements of the points in $\mathbf{X}$. The displaced process has intensity

$$
\rho^{*}(u)=\int \rho(v) p_{v}(u-v) \mathrm{d} v
$$

provided $\rho^{*}$ is locally integrable. If $\mathbf{X}$ is stationary and $p_{u}=p$ does not depend on $u$, then $\mathbf{X}^{*}$ is stationary with intensity $\rho^{*}=\rho$.

Third, the superposition of two point processes $\mathbf{X}$ and $\mathbf{Y}$ is their union $\mathbf{X} \cup \mathbf{Y}$. Assuming the two processes are almost surely disjoint, the intensity of the superposition is given by the sum of intensities of the two processes.

These point process operations may be applied simultaneously. For example, Lund \& Rudemo (2000) considers incomplete observations of tree positions from aerial photography data which are modelled by the combined effects of thinning, random displacements, and superpositioning.

\subsection{The Poisson Process}

The most fundamental point process model is the Poisson process, which for any bounded Borel set $B \subset \mathbb{R}^{d}$ with $\mu(B)>0$ satisfies that

(i) $N(B)$ is Poisson with mean $\mu(B)$;

(ii) the points in $\mathbf{X}_{B}$ are independent of $N(B)$ and i.i.d. with density proportional to $\rho(u), u \in B$.

This description and the properties below easily extend to the case of a general intensity measure, which is not necessarily absolutely continuous with respect to Lebesgue measure (i.e. when the intensity function is not assumed to exist). Poisson processes are studied in detail in Kingman (1993) and Møller \& Waagepetersen (2003). In the stationary case, we have a homogeneous Poisson process, which is also isotropic.

Realisations may appear to be rather chaotic with large empty space and close pairs of points, even when the Poisson process is homogeneous. Examples of simulated homogeneous and inhomogeneous Poisson point processes are shown in Figure 1, where in the inhomogeneous case (the right plot) there is a decreasing log linear trend in the vertical direction. 
Figure 1. Simulation of homogeneous (left) and inhomogeneous (right) Poisson processes on $W=[0,1] \times[0,0.7]$. In both cases the expected number of points is 150 . For the inhomogeneous Poisson process, $\rho(x, y) \propto \exp (-10.6 y),(x, y) \in W$.

The Poisson process is a model for "no interaction" or "complete spatial randomness", since $\mathbf{X}_{A}$ and $\mathbf{X}_{B}$ are independent whenever $A, B \subset \mathbb{R}^{d}$ are disjoint. Moreover, $\rho^{(n)}\left(u_{1}, \ldots, u_{n}\right)=\rho\left(u_{1}\right) \cdots \rho\left(u_{n}\right)$ and $g \equiv 1$, reflecting the lack of interaction. Note that another Poisson process results if we make (a) an independent thinning of a Poisson process, or (b) random independent displacements of the points in a Poisson process, or (c) a superposition of independent Poisson processes. The intensity of the resulting Poisson process in (a)-(c) is of course as described in Section 2.2.

It is straightforward to simulate a homogeneous Poisson process within a bounded region $W \subset \mathbb{R}^{d}$, using (i)-(ii) above or other simple constructions (Section 3.2.3 in Møller \& Waagepetersen, 2003). To obtain a realisation of an inhomogeneous Poisson process on $W$, if $\rho(u)$ is bounded by a constant $K$ for all $u \in W$, we can first simulate a homogeneous Poisson process with intensity $K$ on $W$, and second make an independent $\left(\frac{\rho}{K}\right)$-thinning.

Finally, for statistical modelling, when a vector $z(u)=\left(z_{1}(u), \ldots, z_{k}(u)\right)$ of covariates is available, typically a log linear model of the intensity function is considered,

$$
\log \rho(u)=z(u) \beta^{\mathrm{T}}
$$

where $\beta=\left(\beta_{1}, \ldots, \beta_{k}\right)$ is a regression parameter. This is the canonical link function for an inhomogeneous Poisson process (McCullagh \& Nelder, 1989). In the right plot of Figure 1 , we have that $k=2, z_{1} \equiv 1$, and $z_{2}(u)=y$ is the second coordinate of $u=(x, y)$.

\section{General Description of Cox Processes}

One natural extension of the Poisson process is to consider a non-negative stochastic process $\boldsymbol{\Lambda}=(\Lambda(u))_{u \in \mathbb{R}^{d}}$ such that $\mathbf{X}$ conditional on $\boldsymbol{\Lambda}$ is a Poisson 
process with intensity $\boldsymbol{\Lambda}(\mathrm{Cox}, 1955)$. Then we say that $\mathbf{X}$ is a Cox process driven by $\boldsymbol{\Lambda}$. Usually in applications, $\boldsymbol{\Lambda}$ models an unobserved random heterogeneity. Below we review some general results of relevance for the specific Cox process models studied in Section 4, where the properties of the Cox process $\mathbf{X}$ follow immediately from the properties of the Poisson process $\mathbf{X} \mid \boldsymbol{\Lambda}$.

We have that $\mathbf{X}$ is stationary or isotropic if $\boldsymbol{\Lambda}$ is stationary or isotropic, respectively. Moreover,

$$
\rho^{(n)}\left(u_{1}, \ldots, u_{n}\right)=\mathrm{E}\left\{\Lambda\left(u_{1}\right) \cdots \Lambda\left(u_{n}\right)\right\},
$$

so

$$
\rho(u)=\mathrm{E}\{\Lambda(u)\}, \quad g(u, v)=\mathrm{E}\left\{\frac{\Lambda(u) \Lambda(v)}{\rho(u) \rho(v)}\right\} .
$$

Thus two Cox processes driven by $\Lambda(u)$ respective $h(u) \Lambda(u)$, where $h$ is a nonnegative Borel function, share the same pair correlation function. For most specific Cox process models, $g \geqslant 1$, though there exist some exceptions, see Section 4.2. Similarly, for Borel sets $A, B \subset \mathbb{R}^{d}$ such that $N(A)$ and $N(B)$ have finite variance, the covariance

$$
\operatorname{Cov}(N(A), N(B))=\int_{A} \int_{B} \operatorname{Cov}(\Lambda(u), \Lambda(v)) \mathrm{d} u \mathrm{~d} v+\mu(A \cap B)
$$

is usually nonnegative.

The class of Cox processes is invariant under the basic point process operations. Specifically, an independent $\pi$-thinning of $\mathbf{X}$ is a Cox process driven by $\pi(u) \Lambda(u)$. Furthermore, random independent displacements of the points in $\mathbf{X}$ produce a Cox process driven by

$$
\Lambda^{*}(u)=\int \Lambda(u) p_{v}(u-v) \mathrm{d} v,
$$

cf. (1). Finally, the superposition of two Cox processes driven by independent processes $\Lambda_{1}$ and $\Lambda_{2}$ is a Cox process driven by $\Lambda_{1}+\Lambda_{2}$.

\section{Particular Cox Process Models}

\subsection{Mixed Poisson Process}

The simplest case of a Cox process is a mixed Poisson process where $\Lambda(u)$ is equal to the same nonnegative random variable $\Gamma$ for all locations $u \in \mathbb{R}^{d}$. Like Poisson processes, mixed Poisson processes are usually too simplistic models for real data. In the special case where $\Gamma$ is deterministic, the process is simply a homogeneous Poisson process. Another case is when $\Gamma$ is gamma distributed, 
and hence $N(A)$ follows a negative binomial distribution. In the sequel, we assume that $\Gamma$ is not deterministic.

A mixed Poisson process is stationary and isotropic, with intensity $\rho=\mathrm{E} \Gamma$ and pair correlation function $g=\frac{\mathrm{E}\left(\Gamma^{2}\right)}{\rho^{2}}>1$. By $(3)$,

$$
\operatorname{Cov}(N(A), N(B))=|A||B| \operatorname{Var}(\Gamma)+|A \cap B| \rho,
$$

where $|\cdot|$ denotes Lebesgue measure. Consequently, $N(A)$ and $N(B)$ are positively correlated even if $A$ and $B$ are disjoint. Moreover, $\operatorname{Var} N(A)>\operatorname{E}\{N(A)\}$, i.e. the distribution of $N(A)$ is over-dispersed (while for a Poisson process, $\operatorname{Var}\{N(A)\}=\mathrm{E}\{N(A)\})$.

The class of mixed Poisson processes is obviously closed under independent superpositioning, and also $\pi$-thinning provided $\pi(u)$ is constant for all $u \in \mathbb{R}^{d}$. The displaced process $\mathbf{X}^{*}$ is equivalent to $\mathbf{X}$ provided $p_{v}(\cdot)$ does not depend on $v \in \mathbb{R}^{d}$, since then $\Lambda^{*}(u)=\Gamma$, cf. (4).

Simulation of the process within a bounded region $W \subset \mathbb{R}^{d}$ is just a matter of simulating first the random variable $\Gamma$ and second the homogeneous Poisson process $\mathbf{X}_{W} \mid \Gamma$.

Conversely, for statistical inference, if we observe $\mathbf{X}_{W}$, the conditional distribution of $\Gamma$ given $\mathbf{X}_{W}$ is of interest. This conditional distribution depends on $\mathbf{X}_{W}$ only through $N(W)$, since $N(W)$ is a sufficient statistic for the Poisson process $\mathbf{X}_{W} \mid \Gamma$. It is well known that if $\Gamma$ is gamma distributed, then $\Gamma \mid N(W)$ is gamma distributed.

\subsection{Log Gaussian Cox Process}

Suppose that

$$
\log \Lambda(u)=\Psi(u), \quad u \in \mathbb{R}^{d}
$$

where $\boldsymbol{\Psi}=(\Psi(u))_{u \in \mathbb{R}^{d}}$ is a Gaussian process with mean $\xi(u)=\mathrm{E}\{\Psi(u)\}$ and covariance function $C(u, v)=\operatorname{Cov}(\Psi(u), \Psi(v))$. Then we call $\mathbf{X}$ a $\log$ Gaussian Cox process ( $L G C P$ ) (Møller et al., 1998). To ensure local integrability of $\Lambda(u)$, $C$ has to satisfy certain mild conditions, which are satisfied for covariance models used in practice. Spatio-temporal extensions of log Gaussian Cox processes are studied in Brix \& Diggle (2001) and Brix \& Møller (2001).

As an extension of the $\log$ linear model (2), we may have that $\xi(u)=$ $z(u) \beta^{\mathrm{T}}$. Note that the LGCP is stationary if and only if we can take $\xi(u)$ to be constant and $C(u, v)=C(u-v)$, and it is moreover isotropic if and only if we can take $C(u)=C(\|u\|)$. Figure 2 shows simulations of planar LGCP's within $[0,1]^{2}$, with $\xi(u)=4.10$ and exponential covariance function $C(u, v)=\exp \left(\frac{-\|u-v\|}{0.14}\right)$ (left plot) or Gaussian covariance function $C(u, v)=$ $\exp \left(\frac{-\|u-v\|^{2}}{0.01}\right)$ (right plot). The associated simulated intensity functions are 
also shown, where $\boldsymbol{\Psi}$ is smoother for the Gaussian correlation function (Adler, 1981). Further examples of simulated LGCP's are given in Møller et al. (1998).

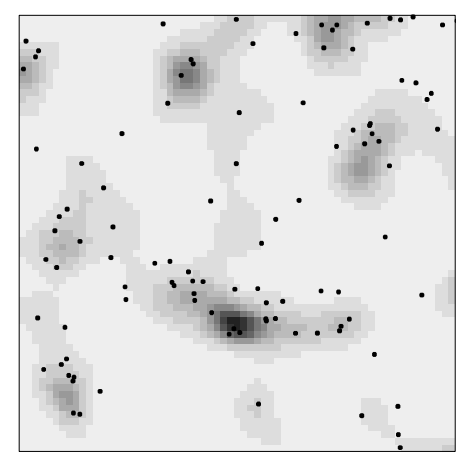

Figure 2. Simulations of LGCP's with exponential (left) and Gaussian (right) correlation functions. The associated simulated random intensity functions are shown in grey scale.

The product densities of a LGCP are particular tractable, as

$$
\log \rho(u)=\xi(u)+\frac{c(u, u)}{2}, \quad g(u, v)=\exp (C(u, v))
$$

and higher-order product densities are nicely expressed in terms of $\rho$ and $g$,

$$
\rho^{(n)}\left(u_{1}, \ldots, u_{n}\right)=\prod_{i=1}^{n} \rho\left(u_{i}\right) \prod_{1 \leqslant i<j \leqslant n} g\left(u_{i}, u_{j}\right) .
$$

Thus $g$ and $C$ are in a one-to-one correspondence, and the distribution of $\mathbf{X}$ is determined by specifying $\xi$ and $C$ or equivalently $\rho$ and $g$. Usually, $C \geqslant 0$ so that $g \geqslant 1$, but there do exist covariance function which can be negative, making it possible that $g(u, v)<1$, cf. Møller et al. (1998).

Another advantageous property is that if we only observe $\mathbf{X}$ within a region $W \subset \mathbb{R}^{d}$, then $\mathbf{X}_{W}$ is specified by the Gaussian process restricted to $W$. Thus we have no problem with edge effects.

Moreover, the class of LGCP's is closed under independent $\pi$-thinning provided $\pi(\cdot)>0$, since $\{\Psi(u)+\log \pi(u)\}_{u \in \mathbb{R}^{d}}$ is the underlying Gaussian process of the thinned process. On the other hand, the class is not closed under random independent displacements or independent superpositioning.

For simulation of a LGCP within a bounded region $W \subset \mathbb{R}^{d}$, we consider a fine finite partition $\Delta_{i}, i \in I$ of $W$, and approximate the Gaussian process 
$(\Psi(u))_{u \in W}$ by a step function with value $\Psi\left(u_{i}\right)$ within $\Delta_{i}$, where $u_{i}$ is a representative point in $\Delta_{i}$. There are many ways of simulating the Gaussian vector $\left(\Psi\left(u_{i}\right)\right)_{i \in I}$, see e.g. Schlather (1999) and Møller \& Waagepetersen (2003). Given a realisation of $\left(\Psi\left(u_{i}\right)\right)_{i \in I}$, we can simulate the inhomogeneous Poisson process $\mathbf{X} \mid\left(\Psi\left(u_{i}\right)\right)_{i \in I}$ using the independent thinning technique in Section 2.3.

The conditional distribution of $\left(\Psi\left(u_{i}\right)\right) i \in I$ given $\mathbf{X}_{W}$ is complicated, and its density is only known up to proportionality. Simulations may be obtained using Langevin-Hastings algorithms, see Møller et al. (1998) and Møller \& Waagepetersen (2003).

\subsection{Shot Noise Cox Process}

Consider a Poisson process $\boldsymbol{\Phi}$ with points $(c, \gamma) \in \mathbb{R}^{d} \times(0, \infty)$ and intensity function $\zeta(c, \gamma)$, and let $k(c, \cdot)$ be a kernel (i.e. a density for a $d$-dimensional continuous random variable on $\mathbb{R}^{d}$ ) specified for any $c \in \mathbb{R}^{d}$. Assuming

$$
\Lambda(u)=\sum_{(c, \gamma) \in \Phi} \gamma k(c, u), \quad u \in \mathbb{R}^{d}
$$

then $\mathbf{X}$ is a shot noise Cox process (SNCP) (Møller, 2003b). The process is an example of a Poisson cluster process, since $\mathbf{X}$ is distributed as the superposition of independent Poisson processes $\mathbf{X}_{(c, \gamma)}$ with intensity functions $\gamma k(c, \cdot)$, $(c, \gamma) \in \boldsymbol{\Phi}$, where we interpret $\mathbf{X}_{(c, \gamma)}$ as a cluster with centre $c$ and mean number of points $\gamma$. SNCP's provide natural models for seed setting mechanisms causing clustering (Brix \& Chadoeuf, 2002), and they can be extended in various interesting ways, see (Møller \& Torrisi, 2005).

Product densities for SNCP's are in general more clumsy to work with than for LGCP's. The intensity is

$$
\rho(u)=\int_{\mathbb{R}^{d}} \int_{0}^{\infty} \gamma k(c, u) \zeta(c, \gamma) \mathrm{d} c \mathrm{~d} \gamma,
$$

and it can be shown that $g \geqslant 1$. Inhomogeneous versions of SNCP's processes may be obtained by adding to the right hand side of (5) a positive term depending on spatial covariates or using a multiplicative model, for example of the form

$$
\Lambda(u)=\exp \left\{z(u) \beta^{\mathrm{T}}\right\} \sum_{(c, \gamma) \in \boldsymbol{\Phi}} \gamma k(c, u),
$$

(Waagepetersen, 2005). A nice feature of the latter model is that the pair correlation function of $\mathbf{X}$ is the same for (5) and (6), i.e. it does not depend on the parameter $\beta$, cf. Section 2 .

A particular tractable case is a Neyman-Scott process (Neyman \& Scott, 1958), where the centre points form a stationary Poisson process with intensity 
$\kappa$ and the $\gamma$ 's are all equal to a positive parameter $\alpha$. If furthermore $k(c, \cdot)$ is the density of the $d$-dimensional normal distribution $\mathrm{N}_{d}\left(c, \omega^{2} I\right)$ with mean $c$ and independent coordinates with variance $\omega^{2}$, then $\mathbf{X}$ is a Thomas process (Thomas, 1949). The Neyman-Scott process is stationary with intensity $\rho=\alpha \kappa$, and the Thomas process is also isotropic with

$$
g(r)=1+\frac{\exp \left\{\frac{-r^{2}}{4 \omega^{2}}\right\}}{\kappa\left(4 \pi \omega^{2}\right)^{\frac{d}{2}}}, \quad r>0 .
$$

Figure 3 shows a simulation of a planar Thomas process within $[0,1]^{2}$, with $\kappa=10, \alpha=10$, and $\omega^{2}=0.1$.

Figure 3. Simulation of a Thomas process and the associated random intensity functions (in grey scale). The crosses show the cluster centres for the Thomas process.

The subclass of shot noise G Cox processes (SNGCP) (Brix, 1999) has

$$
\zeta(c, \gamma)=\kappa \gamma^{-\alpha-1} \frac{\exp (-\tau \gamma)}{\Gamma(1-\alpha)} .
$$

Here, in order to ensure local integrability of $\Phi$, we assume that the parameters satisfy the constraints $\kappa>0, \alpha<1$, and $\tau>0$. If $\alpha<0$, then the $c$ 's form a stationary Poisson process with intensity $\frac{-\kappa \tau^{\alpha}}{\alpha}$, the $\gamma^{\prime}$ 's are i.i.d. and independent of the $c$ 's, and each $\gamma$ is gamma distributed with shape parameter $-\alpha$ and inverse scale parameter $\tau$. The description of $\boldsymbol{\Phi}$ when $0 \leqslant \alpha<1$ is a bit technical, since the c's do not form a locally finite set, see Møller \& Waagepetersen (2002, 2003) and Møller (2003a, 2003b). For a SNGCP with 
$k(c, u)=k(u-c)$ for all $c, u \in \mathbb{R}^{d}$, we have stationarity and $\rho=\kappa \tau^{\alpha-1}$. If furthermore $k(c, \cdot)$ is the density of $\mathrm{N}_{d}\left(c, \omega^{2} I\right)$, we also have isotropy and $g$ is of the same form as for a Thomas process, replacing $\kappa$ in (7) by $\frac{1-\alpha}{\kappa \tau^{\alpha}}$.

A SNGCP with $\alpha=0$ is the so-called Poisson-gamma process (Wolpert \& Ickstadt, 1998 and Daley \& Vere-Jones, 2003). The left plots in Figure 4 show simulations of planar Poisson-gamma processes within $[0,1]^{2}$, with $k(c, \cdot)$ the density of $\mathrm{N}_{2}(c, 0.001 \mathrm{I})$ and $(\kappa, \tau)$ equal to $(15,0.1)$ (upper left panel) or $(7.5,0.05)$ (lower left panel). The simulations are generated as described later, using a truncated random intensity $\Phi \cap\left(W_{\text {ext }} \times(\epsilon, \infty)\right)$, where $W_{\text {ext }} \supset W$ is an extended window and $\epsilon>0$ is a user-specified parameter. The right plots in Figure 4 show the corresponding realisations of $\Phi \cap\left(W_{\text {ext }} \times(\epsilon, \infty)\right)$. Note that the intensity $\rho=\frac{\kappa}{\tau}$ is the same for the two sets of parameter values. With the small values of $\kappa$ and $\gamma$ we obtain fewer and larger clusters than when the larger values are used.

The class of SNCP's is often closed under the basic operations of point processes. More precisely, a $\pi$-thinned SNCP, where $\pi$ is constant, is obviously a SNCP. Further, a displaced SNCP is itself a SNCP with

$$
\Lambda^{*}(u)=\sum_{(c, \gamma) \in \boldsymbol{\Phi}} \gamma \int k(c, v) p_{v}(u-v) \mathrm{d} v
$$

In the case of normal densities $k(c, \cdot) \sim \mathrm{N}_{d}\left(c, \omega^{2} I\right)$ and $p_{v}(\cdot) \sim \mathrm{N}_{d}\left(0, \sigma^{2} I\right)$, the density $u \mapsto \int k(c, v) p_{v}(u-v) \mathrm{d} v$ reduces to that of $\mathrm{N}_{d}\left(c+v,\left(\omega^{2}+\sigma^{2}\right) I\right)$. Furthermore, the superposition of two SNCP's, specified by independent Poisson processes with intensity functions $\zeta$ respective $\tilde{\zeta}$ and the same kernels $k(\cdot, \cdot)$, is a SNCP specified by the Poisson process with intensity function $\zeta+\tilde{\zeta}$ and the kernels $k(\cdot, \cdot)$.

For the simulation of a SNCP within a bounded region $W \subset \mathbb{R}^{d}$, edge effects may occur since the Poisson process $\boldsymbol{\Phi}$ in (5) may be infinite, and so clusters associated to centre points outside $W$ may generate points of the shot noise Cox process within $W$. There is a perfect simulation algorithm which eliminates this problem (Brix \& Kendall, 2002 and Møller, 2003b). However, from a practical view point, it suffices to use results for the error made when $\Phi$ is replaced by $\Phi_{\text {trunc }}=\Phi \cap\left[W_{\text {ext }} \times(\epsilon, \infty)\right]$, where $W_{\text {ext }} \supseteq W$ and $\epsilon \geqslant 0$, see Møller (2003b) and Møller \& Waagepetersen (2003). For example, in Figure 4 where $W=[0,1]^{2}$, we use the truncation $\epsilon=0.0001$ and the extended window $W_{\text {ext }}=[-0.25,1.25]^{2}$.

Finally, conditional simulation of $\Phi_{\text {trunc }}$ given $\mathbf{X}_{W}$ is also discussed in Møller (2003b) and Møller \& Waagepetersen (2003). 

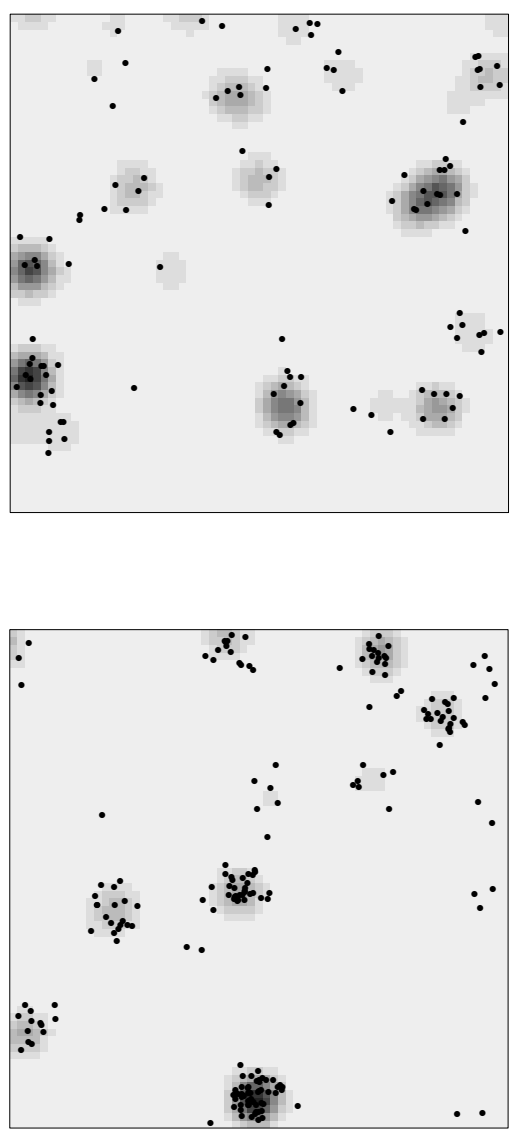

Figure 4. Left: simulations of Poisson-gamma processes (see text for details). Right: corresponding realisations of $\Phi \cap\left(W_{\text {ext }} \times(\epsilon, \infty)\right)$ (only points $(c, \gamma)$ with $\gamma>0.02$ are shown). 


\subsection{Permanent Process}

Consider a bounded Borel set $W \subset \mathbb{R}^{d}$, and let $\nu$ denote the distribution of the homogeneous Poisson process with intensity one. The density of a Cox process restricted to $W$ and with respect to $\nu$ is

$$
f(\mathbf{x})=\mathrm{e}^{|W|} \mathrm{E}\left\{\mathrm{e}^{-\int_{W} \Lambda(u) \mathrm{d} u} \prod_{u \in \mathbf{x}} \Lambda(u)\right\},
$$

for finite subsets $\mathbf{x}$ of $W$. In general this density is not of "known" form. One exception is a mixed Poisson process driven by a gamma distributed random intensity, but as noticed this model is rather uninteresting for real applications. Recently, McCullagh \& Møller (2005) introduced a large model class of point process models, the permanent process, where both the density of the process and the product densities are of a known form. This section considers the subclass of permanent processes, which are also Cox processes.

The driving process of such a Cox process is defined for any positive integer $k$ and real covariance function $C(u, v), u, v \in \mathbb{R}^{d}$ by

$$
\Lambda(u)=\Psi_{1}(u)^{2}+\ldots+\Psi_{k}(u)^{2}, \quad u \in \mathbb{R}^{d}
$$

where $\left(\Psi_{j}(u)\right)_{u \in \mathbb{R}^{d}}, j=1, \ldots, k$ are independent zero-mean Gaussian processes with covariance function $\frac{C}{2}$. As we shall see, both product densities and the density of $\mathbf{X}_{W}$ are expressed in terms of a weighted matrix permanent, explaining why $\mathbf{X}$ is called a permanent Cox process with parameters $\alpha=\frac{k}{2}$ and $C$. The boson (or photon) process (Macchi (1971, 1975), Grandell, 1976 and Daley \& Vere-Jones, 2003) corresponds to $\alpha=1$. Another special case is the mixed Poisson process obtained when $C(u, v)=c$ is constant and hence $\Lambda(u) \sim\left(\frac{c}{2}\right) \chi^{2}(k)$ does not depend on $u \in \mathbb{R}^{d}$.

Clearly, the permanent Cox process is stationary if and only if we can take $C(u, v)=C(u-v)$, and it is moreover isotropic if and only if we can take $C(u, v)=C(\|u-v\|)$. Figure 5 shows simulations of planar permanent Cox processes within $[0,1]^{2}$, with $\alpha=\frac{1}{2}$ and exponential covariance function $C(u, v)=300 \exp \left\{\frac{-\|u-v\|}{0.14}\right\}$ (left plot) or Gaussian covariance function $C(u, v)=300 \exp \left\{\frac{-\|u-v\|^{2}}{0.01}\right\}$ (right plot). The associated simulated intensity functions are also shown.

The following results are verified in McCullagh \& Møller (2005), but first we need to introduce some notation. For any points $x_{1}, \ldots, x_{n} \in \mathbb{R}^{d}$, the symbol $[C]\left(x_{1}, \ldots, x_{n}\right)$ denotes the $n \times n$ matrix with entries $C\left(x_{i}, x_{j}\right)$. The $\alpha$-weighted permanent is

$$
\operatorname{per}_{\alpha}[C]\left(x_{1}, \ldots, x_{n}\right)=\sum_{\sigma} \alpha^{\# \sigma} C\left(x_{1}, x_{\sigma(1)}\right) \cdots C\left(x_{n}, x_{\sigma(n)}\right),
$$



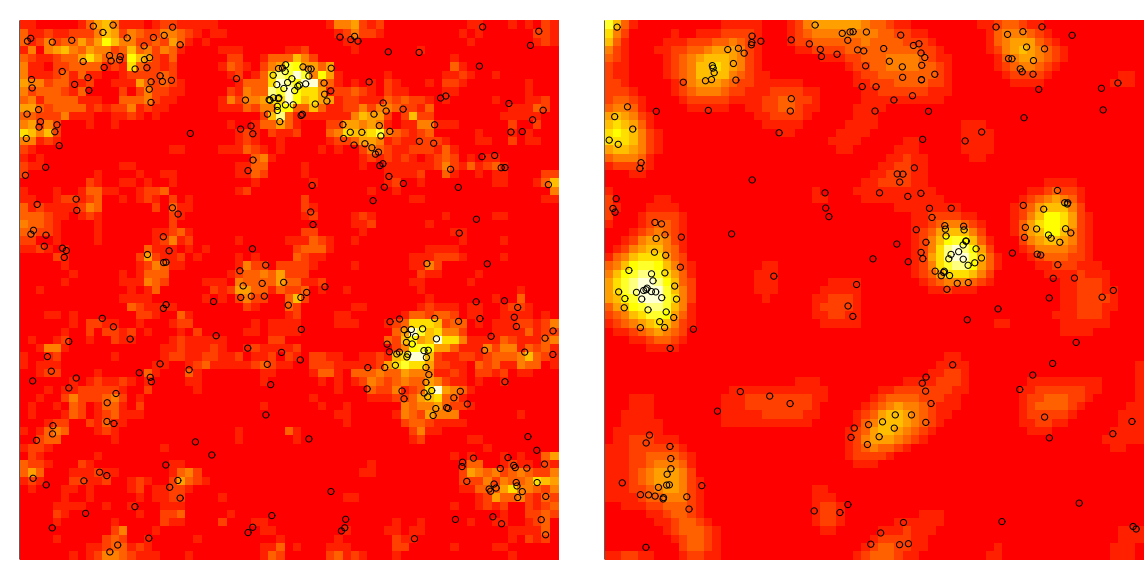

Figure 5. Simulations of permanent Cox processes with exponential (left) and Gaussian (right) correlation functions. The associated simulated random intensity functions are shown in grey scale.

where the sum is over all permutations of $1, \ldots, n$ and $\# \sigma$ is the number of cycles. The usual permanent corresponds to $\alpha=1$ (Minc 1978). Weighted permanents are symmetric functions, and it is often convenient just to write $\operatorname{per}_{\alpha}[C](\mathbf{x})$ for a finite set $\mathbf{x}=\left\{x_{1}, \ldots, x_{n}\right\} \subset \mathbb{R}^{d}$ of $n$ points. Moreover, we set $\operatorname{per}_{\alpha}[C](\emptyset)=1$.

We have

$$
\rho^{(n)}\left(u_{1}, \ldots, u_{n}\right)=\operatorname{per}_{\alpha}[C]\left(u_{1}, \ldots, u_{n}\right) .
$$

Thus

$$
\rho(u)=\alpha C(u, u), \quad g(u, v)=1+\frac{\operatorname{cor}(u, v)^{2}}{\alpha},
$$

where $\operatorname{cor}(u, v)=\frac{C(u, v)}{\{C(u, u) C(v, v)\}^{\frac{1}{2}}}$ is the correlation function. Consequently, $g \geqslant 1$. In particular, $g \rightarrow 1$ as $\alpha \rightarrow \infty$, which is to be expected, since $\mathbf{X}$ can be viewed as the superposition of $k$ independent copies of the permanent process with parameter $k=1$. In some sense the process becomes close to a Poisson process as $\alpha \rightarrow \infty$, since $\frac{\Lambda(u)}{\alpha}$ converges almost surely to $C(u, u)$.

In order to specify the density of $\mathbf{X}_{W}$, we make the following assumptions. Suppose that $W$ is compact and the restriction of the covariance function $C$ to $W \times W$ is a continuous function. Then by Mercer's theorem (e.g. Dieudonné, 1969), the covariance function has spectral representation

$$
C(u, v)=\sum_{r=0}^{\infty} \lambda_{r} e_{r}(u) e_{r}(v), \quad u, v \in W
$$


with absolutely and uniformly convergence in $W \times W$. Here the eigenvalues satisfy $\lambda_{0} \geqslant \lambda_{1} \geqslant \ldots \geqslant 0$, and the eigenfunctions $e_{r}$ form an orthonormal basis of $\mathcal{L}_{2}(W)$, the space of square integrable real Borel functions on $W$ with the inner product $\langle p, q\rangle=\int_{W} p(u) q(u) \mathrm{d} u$. We can therefore take

$$
\Psi_{j}(u)=\sum_{r=0}^{\infty} V_{j, r} e_{r}(u), \quad u \in W
$$

where the $V_{j, r}$ are independent $\mathrm{N}\left(0, \frac{\lambda_{r}}{2}\right)$-distributed random variables. It is convenient to introduce

$$
\tilde{\lambda}_{r}=\frac{\lambda_{r}}{\left(1+\lambda_{r}\right)}, \quad r=0,1, \ldots
$$

and

$$
\tilde{C}(u, v)=\sum_{r=0}^{\infty} \tilde{\lambda}_{r} e_{r}(u) e_{r}(v), \quad u, v \in W
$$

which in fact is a well-defined and continuous covariance function. Further, define $(\tilde{\Lambda}(u))_{u \in W}$ and $\tilde{\mathbf{X}}_{W}$ in the same way as above for $(\Lambda(u))_{u \in W}$ and $\mathbf{X}_{W}$ except that we replace $C$ by $\tilde{C}$. Furthermore,

$$
D \equiv \sum_{r=0}^{\infty} \log \left(1+\lambda_{r}\right)=-\sum_{r=0}^{\infty} \log \left(1-\tilde{\lambda}_{r}\right)
$$

is well-defined, since $0 \leqslant \log \left(1+\lambda_{r}\right) \leqslant \lambda_{r}$ and $\sum \lambda_{r}<\infty$. Then $\mathbf{X}_{W}$ has density

$$
f(\mathbf{x})=\mathrm{e}^{|W|-\alpha D} \operatorname{per}_{\alpha}[\tilde{C}](\mathbf{x}) .
$$

Many other appealing properties are established in McCullagh \& Møller (2005). In particular, the density (11) can be extended to the noninteger case of $2 \alpha>0$ and the case where $C$ is defined in terms of a nonnegative symmetric function $\tilde{C}$ in a somewhat similar way as above (such extensions seem not to be Cox processes). For the extended process, the product densities are still of the form (9).

Moreover, as for LGCP's, edge effects are not a problem, at least for permanent Cox processes, cf. (8). From (8) we also obtain that the class of permanent Cox processes is closed under independent thinning and superpositioning. Specifically, an independent $\pi$-thinning is a permanent process with parameters $\alpha$ and $\sqrt{\pi(u) \pi(v)} \frac{C(u, v)}{2}$. Further, the superposition of two permanent processes with parameters $\alpha$ respective $\alpha^{\prime}$ but the same $C$ is a permanent process with parameters $\alpha+\alpha^{\prime}$ and $C$. On the other hand, random independent 
displacements of the points in a permanent process create a more complicated point process.

Simulation of the permanent Cox process within $W$ is straightforward once we have simulated the Gaussian processes in (8), e.g. by using (10). To the best of my knowledge, algorithms for simulation from $\Lambda(u)_{u \in W} \mid \mathbf{X}_{W}$ have yet not been investigated. Finally, for the computational problem of calculating permanents, we refer to McCullagh \& Møller (2005) and the references therein.

\section{Acknowledgments}

I am grateful to Professor Mohammad Q. Vahidi-Asl for his kind invitation of submitting this paper to the Journal of Statistical Reseach of Iran. My colleague Rasmus Waagepetersen is acknowledged for providing the software for the simulations. This research is funded by the Danish Natural Science Research Council.

\section{References}

Adler, R. (1981). The Geometry of Random Fields, Wiley, New York.

Baddeley, A; Gregori, P.; Mateu, J.; Stoica, R.; Stoyan, D. (2006). Case Studies in Spatial Point Process Modeling. Springer Lecture Notes in Statistics 185, Springer-Verlag, New York.

Baddeley, A.; Møller, J.; Waagepetersen, R. (2000). Non- and semi-parametric estimation of interaction in inhomogeneous point patterns. Statist. Neerlandica 54, 329-350.

Baddeley, A.; Turner, R.; Møller, J.; Hazelton, M. (2005). Residual analysis for spatial point processes (with discussion), J. Roy. Statist. Soc. Ser. B 67, 617-666.

Brix, A. (1999). Generalized gamma measures and shot-noise Cox processes. Adv. Appl. Probab. 31, 929-953.

Brix, A.; Chadoeuf, J. (2002). Spatio-temporal modeling of weeds and shot-noise G Cox processes, Biol. J. 44, 83-99.

Brix, A.; Diggle, P.J. (2001). Spatio-temporal prediction for log-Gaussian Cox processes. J. Roy. Statist. Soc. Ser. B 63, 823-841.

Brix, A.; Kendall, W.S. (2002). Simulation of cluster point processes without edge effects. Adv. Appl. Probab. 34, 267-280.

Brix, A.; Møller, J. (2001). Space-time multitype log Gaussian Cox processes with a view to modelling weed data. Scand. J. Statist. 28, 471-488. 
Cox, D.R. (1955). Some statistical models related with series of events, J. Roy. Statist. Soc. Ser. $B$ 17, 129-164.

Daley, D.J.; Vere-Jones, D. (2003). An Introduction to the Theory of Point Processes. Volume I: Elementary Theory and Methods. Springer-Verlag, New York. Second edition.

Dieudonné, J. (1969). Foundations of Modern Anlysis. Academic Press, NewYork.

Diggle, P.J. (2003). Statistical Analysis of Spatial Point Patterns. Arnold, London, second edition.

Grandell, J. (1976). Doubly stochastic poisson processes. Springer Lecture Notes in Mathematics 529. Springer-Verlag, Berlin.

Kingman, J.F.C. (1993). Poisson Processes. Clarendon Press, Oxford.

Lieshout, M.N.M. van. (2000). Marcov Point Processes and their Applications. Imperical College Press, London.

Lund, J.; Rudemo, M. (2000). Models for point processes observed with noise. Biometrika 87, 235-249.

Macchi, O. (1971). Distribution statistique des instants d'mission des photolectrons d'une lumire thermique. C. R. Acad. Sci. Paris Ser. A 272, 437-440.

Macchi, O. (1975). The coincidence approach to stochastic point processes. Adv. Appl. Probab. 7, 83-122.

McCullagh, P.; Nelder, J. (1989). Generalized Linear Models. Chapman \& Hall, London.

McCullagh, P.; Møller, J. (2005). The permanent process. Tech. Rep. \#29, Department of Mathematical Sciences, Aalborg University.

Minc, H. (1978). Permanents. Addison-Wesley, Reading, MA.

Møller, J. (2003a). A comparison of spatial point process models in epidemiological applications. In Highly Structured Stochastic Systems, P.J. Green, N.L. Hjort and S. Richardson, eds. Oxford University Press, Oxford. pp. 264-268.

Møller, J. (2003b). Shot noise Cox processes. Adv. Appl. Probab. 35, 614-640.

Møller, J.; Syversveen, A.R.; Waagepetersen, P.R. (1998). Log Gaussian Cox processes. Scand. J. Statist. 25, 451-482.

Møller, J.; Torrisi, G.L. (2005). Generalised shot noise Cox processes. Adv. Appl. Probab. 37, $48-74$.

Møller, J.; Waagepetersen, R.P. (2002). Statistical inference for Cox processes. In Spatial Cluster Modelling, A.B. Lawson and D. Denison, eds. Chapman and Hall/CRC, Boca Raton, pp. $37-60$.

Møller, J.; Waagepetersen, R.P. (2003). Statistical Inference and Simulation for Spatial Point Processes. Chapman and Hall/CRC, Boca Raton. 
Møller, J.; Waagepetersen, R.P. (2006). Modern spatial point process modelling and inference. Scand. J. Statist., Tech. Rep. \#12, Department of Mathematical Sciences, Aalborg University. In preparation.

Neyman, J.; Scott, E.L. (1958). Statistical approach to problems of cosmology. J. Roy. Statist. Soc. Ser. B 20, 1-43.

Schlater, M. (1999). Introduction to positive definite functions and unconditional simulation of random fields. Tech. Rep. ST 99-10, Lancaster University.

Stoyan, D.; Kendall W.S.; Mecke, J. (1995). Stochastic Geometry and its Applications. Wiley, Chichester. Second edition.

Thomas, M. (1949). A generalization of Poisson's binomial limit for use in ecology. Biometrika 36, 18-25.

Waagepetersen, R.P. (2005). Discussion of the paper by Baddeley, Turner, Møller \& Hazelton (2005). J. Roy. Statist. Soc. Ser. B. 67 to appear.

Wolpert, R.L.; Ickstadt, K. (1998). Poisson/gamma random field models for spatial statistics. Biometrika 85, 251-267.

| Received: Feb 12, 2006

\section{Jesper Møller}

Fredrik Bajers Vej 7G,

DK-9220 Aalborg $\varnothing$.

e-mail: jm@math.aau.dk 